\title{
The Correlates of Violence against Women in India: Findings from the Recent National Demographic Health Survey
}

\author{
Dinesh Chaurasiya $^{1}$, Vaishali Chaurasia ${ }^{2}$, Shekhar Chauhan ${ }^{3 *}$, Ratna Patel ${ }^{4}$ \\ Ajay Gupta $^{5}$ and Sayeed Unisa ${ }^{6}$ \\ ${ }^{1,2,3,4 \& 5}$ Research Scholar, ${ }^{6}$ Professor, Department of Mathematical Demography \& Statistics, \\ International Institute for Population Sciences, Mumbai, Maharashtra, India \\ *Correspondence Author \\ E-Mail: shekhariips2486@gmail.com
}

\begin{abstract}
Violence against women is a serious human rights abuse and public health issue in India. The Intimate Partner violence (IPV) cases among Indian couples are very high. This article aims to find the determinant of Intimate Partner Violence in India. The data are drawn from the fourth round of National Family Health Survey (NFHS-IV). According to Demographic Health Survey guidelines, IPV is measured using 13-item questions in women questionnaire. This section is analysed to fulfil the objective of the study. Bivariate and multivariate logistic regression is used to find out the unadjusted and adjusted odds ratio. The analysis is carried out using STATA version 14. The prevalence of IPV, emotional violence $(\mathrm{EV})$, physical violence (PV) and sexual violence (SV) is $33.15,13.23,29.68$ and 6.60 respectively. The likelihood of IPV increases with the increase in marital duration. All kind of violence is less likely to occur in rural areas (IPV: $\mathrm{AOR}=0.86$, p<0.01; EV: AOR=0.81, p<0.01; PV: AOR=0.85, p<0.01; \& $\mathrm{SV}: \mathrm{AOR}=0.92, \mathrm{p}=0.09)$. Hindu women are more likely to face all kind of violence than women in other religion. Alcohol consumption is one of the predominant factors for IPV in India $(\mathrm{AOR}=3.08, \mathrm{CI}=2.96-3.21, \mathrm{p}<0.01)$. From this study, we find that marital duration, the age difference of spouses, number of children, place of residence, caste, religion, and education of couple, alcohol consumption and wealth index are some of the important predictors of IPV in India.
\end{abstract}

Keywords: Violence against Women, Emotional Violence, Physical Violence, India, National Family Health Survey-IV

\section{INTRODUCTION}

Intimate Partner Violence (IPV) is found in all the societies around the world (Campbell, 2002; Ali et al., 2011; Silverman et al., 2007; Raj \& Silverman, 2002; Devries et al., 2010, \& Hyderet al., 2007). Many causes and consequences are associated with the occurrence of IPV (Jewkes, 2002; Campbell, 2002; Leonard, 2005; \& Varma et al., 2007). Despite a plethora of literature available to get insight into the causes and consequences of Intimate Partner Violence, the problem persists. Unveiling the different aspects of IPV reveals that this issue in particular needs to be understood in connection with the human rights discourse. IPV is not only associated with health burdens but also has intergenerational effects as well as sociodemographic consequences of physical and sexual abuse (Garcia \& WHO, 2005, Panda \& Agarwal, 2005, and Agarwal \& Panda, 2007). Women who experience violent acts in their households, the environment that is supposed to be the safest place, not only suffer from health-related issues but also carry the emotional and psychological burden. Intimate partner violence can occur at any stage of life and women may also suffer IPV during pregnancy (Bloom et al., 2010; WHO, 2011; Devries, 2010; \& LaRusso, 2016). In this study, we have emphasized upon various predictors that can affect the intimate partner violence. Women often put up with men's violence against them because they see no alternatives.

\section{A. Intimate Partner Violence in Indian Context}

The patriarchal system in India secludes the women from their families after marriage. After marriage, a woman is the responsibility of her husband's family. Moreover, in the ideal setting of the rural family, a woman decidedly has no control over the economic resources. Men are seen as the providers and women as those upon whose is the responsibility of maintaining the code and honour of the family. Intimate Partner Violence not only include violence against women but it also entrenched gender inequality, constitutes an extreme form of discrimination against women and has long-term consequences. Intimate Partner Violence is a problem that occurs in all settings, albeit with a different severity level varies across socio-cultural characteristics (WHO, 2012; \& Bamiwuye, 2014). Understanding the cause of Intimate Partner Violence (IPV) is substantially more difficult in India as women themselves justify the violence against them (Jejeebhoy, 1998). Not only in India, but women around the world also justify violence against them (George \& Rahangdale, 1999; Bui \& Morash, 1999; Perilla, 1999; Schuler et al., 1996; \& Chin, 1994).

\section{DATA AND METHODOLOGY}

\section{A. Data Source and Sample Selection}

Data for this study were drawn from the fourth round of National Family Health Survey 2015-16 (NFHS-IV). This study is a result of a nationally representative probability sample of all the women of reproductive ages (15-49) with a nationwide sample of women selected from 104377 households. In women questionnaire of NFHS IV, there is a special section on Household relation section (module-11) 
which covers questions related to violence against women. Couples having complete information on variables under study were included in the study. In very few cases women were older than their husbands, that cases were excluded from study. The total sample of couples included after filtering out was 60,918 in this study.

\section{B. Variables}

1. Independent Variables: The various independent variables are used for the study. The background characteristics of the women and partner's characteristics are the two main domains covering independent variables. In background characteristics, place of residence, caste, religion, wealth quintile, children ever born, spousal age difference, women's education status, and marital duration were taken as independent variables. Husband's education level and husband's alcohol consumption were taken as independent variable also.

2. Dependent Variables: Our dependent variables are physical, emotional and sexual violence. One more variable is created by adding all these three types of violence named "Intimate Partner Violence." The NFHS IV collected detailed information about different forms of violence. To measure physical, sexual, and emotional violence, questions included are: Does/did your husband ever did any of the following things to you in the past 12 months.

3. Physical Violence: Push you, shake you, or throw something at you, Twist your arm or pull your hair, Slap you, Punch you with his fist or with something that could hurt you, Kick you, drag you or beat you up, Try to choke you or burn you on purpose, Threaten or attack you with a knife, gun, or any other weapon. These questions are used to create the index of physical violence

4. Sexual Violence: Physically force you to have sexual intercourse with him even when you did not want to, Physically force you to perform any other sexual acts you did not want to, Force you with threats or in any other way to perform sexual acts you did not want to. These questions are used to create sexual violence

5. Emotional Violence: Say or do something to humiliate you in front of others, Threaten to hurt you or someone close to you, Insult you or make you feel bad about yourself. These questions are combined to create emotional violence.

\section{Methodology}

Mean age of husband and wife is calculated to get an insight into the spousal age gap difference.

Regression is also used to understand the predictor of all reasons for violence. To observe this, logistic regression is used in which form of violence is categorized as a dichotomous variable. The unadjusted as well as adjusted odds have been applied for a better understanding of the predictors of the violence against women.

\section{RESULTS}

In Table I, the data show that the mean age of husbands is higher than that of their wives across all the domains of violence. The mean age of husbands and wives is $37.84 \pm$ 9.24 and $32.68 \pm 8.02$ years respectively, while the mean spousal age difference is $5.17 \pm 4.16$ years. Mean age of women who did not face intimate partner violence is little higher (5.19 as compared to 5.11 years) than those women who have faced intimate partner violence. For all the domains of violence including intimate partner violence, when women are suffering from violence, the mean of spousal age gap difference is about five years.

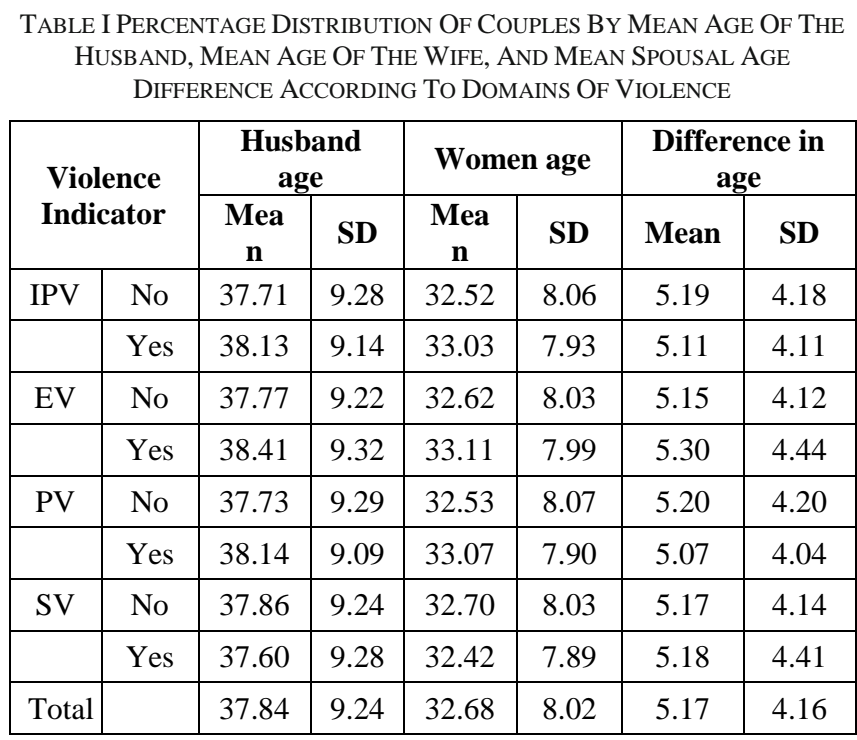

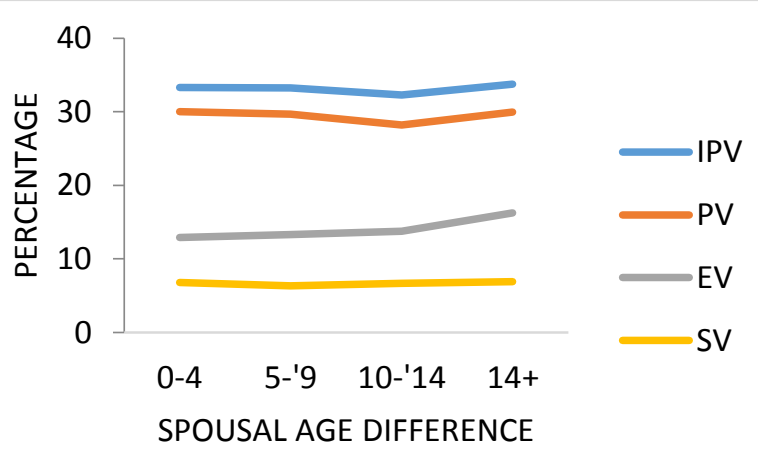

Fig. 1 Prevalence of different forms of Violence by Spousal Age Difference, NHHS-IV India

Figure 1 presents the proportion of women who have experienced Intimate Partner Violence, Emotional Violence, Physical Violence and Sexual violence by the spousal age difference. For emotional violence that had been experienced by women, the proportion increases consistently from $12.9 \%$ among couples with an age difference of $0-4$ years to $16.2 \%$ among those whose age difference was 14 years and more. For Intimate Partner Violence and Emotional violence, across marital duration, the violence experienced ranged from $33.3 \%$ to $33.8 \%$ ( $\mathrm{p}<$ $0.001)$ and $12.9 \%-16.2 \%(\mathrm{p}<0.001)$ respectively. The 
Dinesh Chaurasiya, Vaishali Chaurasia, Shekhar Chauhan, Ratna Patel Ajay Gupta and Sayeed Unisa

figure shows that sexual violence is the least prevalent and around 6.8 to 7 percent of women have faced sexual violence in various spousal age difference categories. The sexual violence goes underreported and non-reported due to various reasons, and this can be one of the causes of the low prevalence of sexual violence among women. The figure presents a general perception that with an increase in spousal age difference the prevalence of any type of violence, including intimate partner violence, increases except physical violence. The prevalence of physical violence remains the same when the spousal age difference is least (0-4 years) and highest (14+ years) with little decline in intermediate spousal age difference group.

TABle II Percentage Distribution Of Women By ExPERIEnCEd Violence TyPes, NATional Family Health SuRVEy-Iv, India

\begin{tabular}{|c|c|c|c|c|c|c|c|c|c|c|c|c|}
\hline 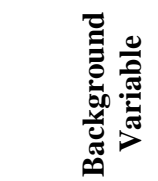 & 它 & $\frac{\grave{\Xi}}{\grave{Z}}$ & 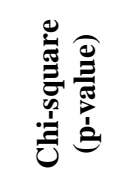 & 怘 & $\frac{\grave{\Xi}}{\grave{\Xi}}$ & 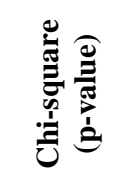 & 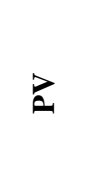 & $\frac{\grave{\Xi}}{\grave{\Xi}}$ & 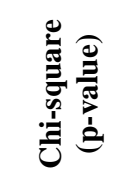 & is & $\frac{\grave{\Xi}}{\grave{\Xi}}$ & 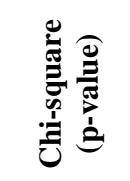 \\
\hline \multicolumn{13}{|c|}{ Children Ever Born } \\
\hline 0 & 21.22 & 1,092 & 1300 & 9.07 & 445 & 288.062 & 17.53 & 898 & 1100 & 5.09 & 272 & 195.2909 \\
\hline $1-2$ & 29.78 & 8,507 & $(<0.001)$ & 12.03 & 3,253 & $(<0.001)$ & 26.43 & 7,468 & $(<0.001)$ & 5.56 & 1,625 & $(<0.001)$ \\
\hline $3-4$ & 39.59 & 6,966 & & 15.51 & 2,695 & & 36.08 & 6,321 & & 8.03 & 1,435 & \\
\hline $4+$ & 45.16 & 2,535 & & 17.48 & 968 & & 41.34 & 2,303 & & 10.03 & 556 & \\
\hline \multicolumn{13}{|c|}{ Spousal Age Difference } \\
\hline $0-4$ & 33.27 & 10,090 & 14.98 & 12.91 & 3,752 & 4.4938 & 30.03 & 9,053 & 25.2508 & 6.79 & 2,072 & 5.1831 \\
\hline $5-9$ & 33.22 & 6,740 & $(0.002)$ & 13.29 & 2,661 & $(0.213)$ & 29.66 & 5,954 & $(<0.001)$ & 6.31 & 1,336 & $(0.159)$ \\
\hline $10-14$ & 32.25 & 1,797 & & 13.75 & 735 & & 28.21 & 1,569 & & 6.65 & 370 & \\
\hline $14+$ & 33.77 & 473 & & 16.24 & 213 & & 29.95 & 414 & & 6.96 & 110 & \\
\hline \multicolumn{13}{|c|}{ Marital Duration } \\
\hline $0-5$ & 22.90 & 2,613 & 573.8847 & 9.21 & 1,004 & 184.225 & 19.66 & 2,207 & 610.98 & 4.95 & 582 & 55.4949 \\
\hline $6-10$ & 33.24 & 3,641 & $(<0.001)$ & 12.82 & 1,376 & $(<0.001)$ & 30.06 & 3,255 & $(<0.001)$ & 6.76 & 734 & $(<0.001)$ \\
\hline $11-15$ & 35.00 & 4,011 & & 13.77 & 1,522 & & 31.72 & 3,612 & & 7.47 & 869 & \\
\hline $15+$ & 36.78 & 8,560 & & 14.91 & 3,342 & & 33.04 & 7,676 & & 6.83 & 1,645 & \\
\hline \multicolumn{13}{|c|}{ Place of Residence } \\
\hline Urban & 27.33 & 4,779 & 238.94 & 11.46 & 1,933 & 35.8428 & 24.08 & 4,204 & 229.8038 & 4.94 & 898 & 75.662 \\
\hline Rural & 36.31 & 14,321 & $(<0.001)$ & 14.19 & 5,428 & $(<0.001)$ & 32.72 & 12,786 & $(<0.001)$ & 7.5 & 2,990 & $(<0.001)$ \\
\hline \multicolumn{13}{|l|}{ Caste } \\
\hline $\mathrm{SC}$ & 40.39 & 4,196 & 773.19 & 16.44 & 1,650 & 287.7492 & 36.81 & 3,795 & 730.0543 & 8.60 & 943 & 188.4117 \\
\hline ST & 37.02 & 3,423 & $(<0.001)$ & 15.19 & 1,330 & $(<0.001)$ & 33.31 & 3,014 & $(<0.001)$ & 8.38 & 728 & $(<0.001)$ \\
\hline $\mathrm{OBC}$ & 34.43 & 7,993 & & 13.48 & 3,017 & & 30.85 & 7,193 & & 6.36 & 1,534 & \\
\hline Other & 24.44 & 2,797 & & 9.48 & 1,018 & & 21.56 & 2,458 & & 4.65 & 536 & \\
\hline \multicolumn{13}{|l|}{ Religion } \\
\hline Hindu & 33.65 & 15,094 & 180.38 & 13.34 & 5,716 & 41.317 & 30.23 & 13,580 & 241.9588 & 6.56 & 3,041 & 14.836 \\
\hline Muslim & 32.19 & 2,238 & $(<0.001)$ & 13.6 & 984 & $(<0.001)$ & 28.13 & 1,909 & $(<0.001)$ & 6.87 & 462 & $(0.001)$ \\
\hline Other & 27.83 & 1,761 & & 10.57 & 656 & & 25.01 & 1,495 & & 6.48 & 385 & \\
\hline \multicolumn{13}{|c|}{ Wealth Index } \\
\hline Poorest & 45.90 & 5,195 & 33.33 & 18.37 & 1,995 & 678.6787 & 42.38 & 4,810 & 2200 & 11.27 & 1,230 & 627.8855 \\
\hline Poorer & 40.48 & 4,749 & $(<0.001)$ & 16.14 & 1,837 & $(<0.001)$ & 36.67 & 4,249 & $(<0.001)$ & 7.93 & 962 & $(<0.001)$ \\
\hline Middle & 35.06 & 3,947 & & 14.03 & 1,563 & & 31.38 & 3,454 & & 6.66 & 785 & \\
\hline Richer & 28.99 & 3,163 & & 11.06 & 1,193 & & 25.73 & 2,763 & & 4.92 & 570 & \\
\hline Richest & 18.63 & 2,046 & & 7.89 & 773 & & 15.52 & 1,714 & & 3.26 & 341 & \\
\hline
\end{tabular}




\begin{tabular}{|c|c|c|c|c|c|c|c|c|c|c|c|c|}
\hline \multicolumn{13}{|c|}{ Women Education Level } \\
\hline No Education & 43.93 & 8,137 & 1900 & 17.79 & 3,170 & 603.5167 & 40.21 & 7,422 & 2000 & 9.13 & 1,719 & 402.1418 \\
\hline Primary & 39.29 & 3,204 & $(<0.001)$ & 15.00 & 1,195 & $(<0.001)$ & 36.05 & 2,914 & $(<0.001)$ & 7.93 & 670 & $(<0.001)$ \\
\hline Secondary & 28.20 & 6,926 & & 11.26 & 2,684 & & 24.53 & 5,970 & & 5.38 & 1,366 & \\
\hline Higher & 14.10 & 833 & & 5.76 & 312 & & 11.88 & 684 & & 2.51 & 133 & \\
\hline \multicolumn{13}{|c|}{ Husband Education Level } \\
\hline No Education & 45.15 & 4,759 & 1600 & 19.11 & 1,915 & 585.3054 & 41.45 & 4,345 & 1600 & 9.71 & 1,041 & 386.9045 \\
\hline Primary & 40.72 & 3,501 & $(<0.001)$ & 15.73 & 1,342 & $(<0.001)$ & 37.01 & 3,199 & $(<0.001)$ & 8.50 & 749 & $(<0.001)$ \\
\hline Secondary & 30.87 & 9,320 & & 12.27 & 3,564 & & 27.39 & 8,174 & & 5.89 & 1,850 & \\
\hline Higher & 17.98 & 1,472 & & 6.47 & 520 & & 15.06 & 1,226 & & 3.11 & 236 & \\
\hline \multicolumn{13}{|c|}{ Husband Drink Alcohol } \\
\hline No & 24.79 & 9,724 & 43000 & 8.93 & 3,413 & 2000 & 21.48 & 8,346 & 4400 & 4.01 & 1,631 & 1400 \\
\hline Yes & 53.65 & 9,376 & $(<0.001)$ & 23.77 & 3,948 & $(<0.001)$ & 49.80 & 8,644 & $(<0.001)$ & 12.96 & 2,257 & $(<0.001)$ \\
\hline
\end{tabular}

The data presented in Table II show the distribution of women by the type of violence they had experienced from their partners in the past. The violence against women is highly associated with the number of children ever born to a woman. 21 percent of women having no children have suffered intimate partner violence as compared to 45 percent of women who had 4 or more children born to them. It suggests that children ever born is a factor in determining the violence against women, it is as such not the children ever born to them but the length of marital duration they are in that decides the violence against them. As the number of children ever born increases, there is an increase in marital duration also, and that inadvertently decides the onset of violence against women. Marital duration is significantly associated with all the domains of Violence. Women with 05 years of marital duration, experienced less violence as compared to women with higher marital duration. Physical Violence across marital duration ranges from $19.7 \%$ in $0-5$ years of marital duration to $33.0 \%$ in women who were in more than 15 years of marital union $(\mathrm{p}<0.001)$.

The spousal age difference is found to be statistically associated with the IPV and physical violence but not with the emotional violence and sexual violence. A higher percentage of women in rural areas face violence against them as compared to their counterparts. Religion and caste are significantly associated with all the domains of violence. Further, data shows that all the domains of violence are significantly associated with household wealth. Among all the wealth quintile, richest women experience less violence. The prevalence of IPV is $43.9 \%, 39.3 \%, 28.2 \%$ and $14.1 \%$ among those with no education, primary, secondary and higher education respectively. Education, women's as well as her husband's, is a barrier to the onset of violence against women as an increase in educational status significantly decreases the chances of violence against women. The percentage of women who reported that they had experienced violence from their partner is strikingly higher among spouses of men who consumed alcohol. The husband's alcohol consumption significantly raises the chance of a high rate of violence against women. For all the three domains of violence, the result shows that women whose husband consumes alcohol are more prone to face violence against them as their counterparts.

The data presented in Table III show the results of a bivariate logistic regression that examines the relationship between IPV domains and socioeconomic characteristics. For intimate partner violence, the odds reduce consistently, as the spousal age difference increases.

The likelihood of emotional violence increases with the increase in marital duration. The unadjusted odds of facing any type of violence are higher among rural women than in urban women, but adjusted odds are lower in rural women than their counterparts. Without adjusting the other characteristics, rural women are 1.4 times more likely to face intimate partner violence but when adjusted for education, wealth index, and other background characteristics the odds (IPV: AOR=0.86, p<0.01; EV: AOR $=0.81, \quad \mathrm{p}<0.01 ; \quad \mathrm{PV}: \quad \mathrm{AOR}=0.85, \mathrm{p}<0.01 ; \quad \& \quad \mathrm{SV}$ : $\mathrm{AOR}=0.92$ ) of facing violence significantly decreases for rural women.

The adjusted odds ratio of facing any form of violence is higher among Muslim women than in women belong to Hindu religion. Muslim women are 1.1 times more likely to face intimate partner violence than women in Hindu religion. Similarly, Muslim women are 1.3 times, 1.1 times, and 1.3 times are more likely to face emotional $(\mathrm{AOR}=1.32$, $\mathrm{CI}=1.20-1.44, \mathrm{p}<0.01)$, physical $(\mathrm{AOR}=1.10, \mathrm{CI}=1.03-1.18$, $\mathrm{p}<0.01)$, and sexual violence $(\mathrm{AOR}=1.33, \mathrm{CI}=1.17-1.50$, $\mathrm{p}<0.01)$ respectively as compared to women in Hindu religion.

Wealth index is one of the most critical factors in determining the violence against women. Both in adjusted as well as unadjusted odds ratio, the women in poorest wealth quintile are more likely to face any form of violence than women in subsequent well off quintile. As a woman progresses to a richer wealth quintile, she subsequently decreases the likelihood of facing with any form of 
Dinesh Chaurasiya, Vaishali Chaurasia, Shekhar Chauhan, Ratna Patel Ajay Gupta and Sayeed Unisa

violence. Similarly as the higher wealth index, higher education also means that a woman faces less violence as compared to a woman who is having less education. Not only women's education but also the husband's education matters when it comes to defining the violence against women.

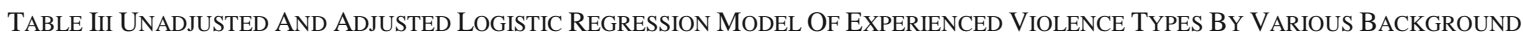
CHARACTERISTICS, NATIONAL FAMILY HEALTH SURVEY-IV, INDIA

\begin{tabular}{|c|c|c|c|c|c|c|c|c|c|c|c|c|c|c|c|c|}
\hline \multirow{3}{*}{ 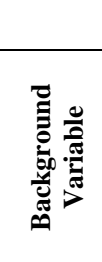 } & \multicolumn{4}{|c|}{ IPV } & \multicolumn{4}{|c|}{$\mathbf{E V}$} & \multicolumn{4}{|c|}{ PV } & \multicolumn{4}{|c|}{ SV } \\
\hline & \multirow[t]{2}{*}{ ڤี } & \multirow[t]{2}{*}{$\frac{0}{0}$} & \multicolumn{2}{|c|}{ 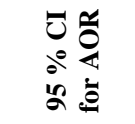 } & \multirow[t]{2}{*}{ 气ै } & \multirow[t]{2}{*}{$\frac{2}{0}$} & \multicolumn{2}{|c|}{ 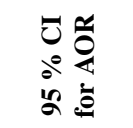 } & \multirow[t]{2}{*}{ 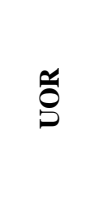 } & \multirow[t]{2}{*}{ 旁 } & \multicolumn{2}{|c|}{ 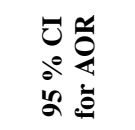 } & \multirow[t]{2}{*}{ 응 } & \multirow[t]{2}{*}{ 竜 } & \multicolumn{2}{|c|}{ 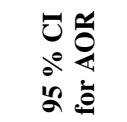 } \\
\hline & & & ـ & $\triangleright$ & & & ユ & $\triangleright$ & & & ـ & $\triangleright$ & & & ـ & $\triangleright$ \\
\hline \multicolumn{17}{|c|}{ Children Ever Born } \\
\hline \multicolumn{17}{|l|}{0 ( } \\
\hline $1-2$ & $\begin{array}{c}1.45 * * \\
*\end{array}$ & $\begin{array}{c}1.35 * * \\
*\end{array}$ & $\begin{array}{c}1.2 \\
4\end{array}$ & $\begin{array}{c}1.4 \\
6\end{array}$ & $\begin{array}{c}1.27 * * \\
*\end{array}$ & $\begin{array}{c}1.17 * * \\
*\end{array}$ & $\begin{array}{c}1.0 \\
4\end{array}$ & $\begin{array}{c}1.3 \\
1\end{array}$ & $\begin{array}{c}1.55^{* *} \\
*\end{array}$ & $\begin{array}{c}1.42^{* *} \\
*\end{array}$ & $\begin{array}{c}1.3 \\
0\end{array}$ & $\begin{array}{c}1.5 \\
5\end{array}$ & 1.02 & $1.01 *$ & $\begin{array}{c}0.8 \\
7\end{array}$ & $\begin{array}{c}1.1 \\
7\end{array}$ \\
\hline $3-4$ & $\begin{array}{c}2.15 * * \\
*\end{array}$ & $\begin{array}{c}1.48 * * \\
*\end{array}$ & $\begin{array}{c}1.3 \\
5 \\
\end{array}$ & $\begin{array}{c}1.6 \\
2\end{array}$ & $\begin{array}{c}1.75^{* *} \\
*\end{array}$ & $\begin{array}{c}1.23^{* *} * \\
*\end{array}$ & $\begin{array}{c}1.0 \\
9\end{array}$ & $\begin{array}{c}1.4 \\
0 \\
\end{array}$ & $\begin{array}{c}2.36^{* *} \\
*\end{array}$ & $\begin{array}{c}1.59 * * \\
*\end{array}$ & $\begin{array}{c}1.4 \\
4\end{array}$ & $\begin{array}{c}1.7 \\
5\end{array}$ & $\begin{array}{c}1.47 * * \\
*\end{array}$ & 1.17 & $\begin{array}{c}0.9 \\
9\end{array}$ & $\begin{array}{c}1.3 \\
7 \\
\end{array}$ \\
\hline $4+$ & $\begin{array}{c}2.77 * * \\
* \\
\end{array}$ & $\begin{array}{c}1.62 * * \\
*\end{array}$ & $\begin{array}{c}1.4 \\
6 \\
\end{array}$ & $\begin{array}{c}1.8 \\
0 \\
\end{array}$ & $\begin{array}{c}2.06^{* * *} \\
* \\
\end{array}$ & $\begin{array}{c}1.25^{* *} \\
* \\
\end{array}$ & $\begin{array}{c}1.0 \\
8 \\
\end{array}$ & $\begin{array}{c}1.4 \\
4 \\
\end{array}$ & $\begin{array}{c}3.00^{* *} \\
*\end{array}$ & $\begin{array}{c}1.71^{* * *} \\
*\end{array}$ & $\begin{array}{c}1.5 \\
3 \\
\end{array}$ & $\begin{array}{c}1.9 \\
1 \\
\end{array}$ & $\begin{array}{c}1.86^{* *} \\
* \\
\end{array}$ & 1.29 & $\begin{array}{c}1.0 \\
7 \\
\end{array}$ & $\begin{array}{c}1.5 \\
5 \\
\end{array}$ \\
\hline $10-14$ & $\begin{array}{c}0.90 * * \\
*\end{array}$ & $0.94 * *$ & $\begin{array}{c}0.8 \\
8 \\
\end{array}$ & $\begin{array}{c}1.0 \\
1 \\
\end{array}$ & 1.03 & $1.09 *$ & $\begin{array}{c}0.9 \\
9 \\
\end{array}$ & $\begin{array}{c}1.1 \\
9 \\
\end{array}$ & $\begin{array}{c}0.88^{* * *} \\
*\end{array}$ & $\begin{array}{c}0.91 * * \\
*\end{array}$ & $\begin{array}{c}0.8 \\
5 \\
\end{array}$ & $\begin{array}{c}0.9 \\
7 \\
\end{array}$ & 0.93 & 1.04 & $\begin{array}{c}0.9 \\
2 \\
\end{array}$ & $\begin{array}{c}1.1 \\
7 \\
\end{array}$ \\
\hline $14+$ & $0.91 *$ & 0.91 & $\begin{array}{c}0.8 \\
1 \\
\end{array}$ & $\begin{array}{c}1.0 \\
3 \\
\end{array}$ & $1.15^{*}$ & $1.19 * *$ & $\begin{array}{c}1.0 \\
2 \\
\end{array}$ & $\begin{array}{c}1.4 \\
0 \\
\end{array}$ & $0.88 * *$ & $0.87 * *$ & $\begin{array}{c}0.7 \\
7 \\
\end{array}$ & $\begin{array}{c}0.9 \\
9 \\
\end{array}$ & 1.06 & 1.10 & $\begin{array}{c}0.8 \\
8\end{array}$ & $\begin{array}{c}1.3 \\
6\end{array}$ \\
\hline \multicolumn{17}{|c|}{ Marital Duration } \\
\hline \multicolumn{17}{|l|}{$0-5$ ß } \\
\hline $6-10$ & $\begin{array}{c}1.54^{* *} \\
*\end{array}$ & $\begin{array}{c}1.22 * * \\
*\end{array}$ & $\begin{array}{c}1.1 \\
4\end{array}$ & $\begin{array}{c}1.3 \\
0\end{array}$ & $\begin{array}{c}1.39 * * \\
*\end{array}$ & $\begin{array}{c}1.16^{* *} \\
*\end{array}$ & $\begin{array}{c}1.0 \\
5\end{array}$ & $\begin{array}{c}1.2 \\
7\end{array}$ & $\begin{array}{c}1.62 * * \\
*\end{array}$ & $\begin{array}{c}1.25^{* *} \\
*\end{array}$ & $\begin{array}{l}1.1 \\
7\end{array}$ & $\begin{array}{c}1.3 \\
5\end{array}$ & $\begin{array}{c}1.26^{* *} \\
*\end{array}$ & 1.02 & $\begin{array}{c}0.9 \\
0\end{array}$ & $\begin{array}{c}1.1 \\
6\end{array}$ \\
\hline $11-15$ & $\begin{array}{c}1.73^{* *} \\
* \\
\end{array}$ & $\begin{array}{c}1.27 * * \\
* \\
\end{array}$ & $\begin{array}{c}1.1 \\
8 \\
\end{array}$ & $\begin{array}{c}1.3 \\
6 \\
\end{array}$ & $\begin{array}{c}1.54 * * \\
*\end{array}$ & $\begin{array}{c}1.20 * * \\
*\end{array}$ & $\begin{array}{c}1.0 \\
8 \\
\end{array}$ & $\begin{array}{c}1.3 \\
2 \\
\end{array}$ & $\begin{array}{c}1.84 * * \\
* \\
\end{array}$ & $\begin{array}{c}1.31 * * \\
* \\
\end{array}$ & $\begin{array}{c}1.2 \\
2 \\
\end{array}$ & $\begin{array}{c}1.4 \\
1 \\
\end{array}$ & $\begin{array}{c}1.48^{* *} * \\
*\end{array}$ & 1.10 & $\begin{array}{c}0.9 \\
7\end{array}$ & $\begin{array}{c}1.2 \\
5\end{array}$ \\
\hline $15+$ & $\begin{array}{c}1.83^{* *} \\
* \\
\end{array}$ & $\begin{array}{c}1.24 * * \\
*\end{array}$ & $\begin{array}{c}1.1 \\
6 \\
\end{array}$ & $\begin{array}{c}1.3 \\
2 \\
\end{array}$ & $\begin{array}{c}1.65^{* * *} \\
*\end{array}$ & $\begin{array}{c}1.24 * * \\
*\end{array}$ & $\begin{array}{c}1.1 \\
3 \\
\end{array}$ & $\begin{array}{c}1.3 \\
6 \\
\end{array}$ & $\begin{array}{c}1.92^{* *} \\
*\end{array}$ & $\begin{array}{c}1.25^{* *} \\
* \\
\end{array}$ & $\begin{array}{c}1.1 \\
7 \\
\end{array}$ & $\begin{array}{c}1.3 \\
4 \\
\end{array}$ & $\begin{array}{c}1.35^{* *} \\
*\end{array}$ & 0.94 & $\begin{array}{c}0.8 \\
3 \\
\end{array}$ & $\begin{array}{c}1.0 \\
6 \\
\end{array}$ \\
\hline ST & $\begin{array}{c}0.74 * * \\
*\end{array}$ & $\begin{array}{c}0.66^{* *} \\
*\end{array}$ & $\begin{array}{c}0.6 \\
2 \\
\end{array}$ & $\begin{array}{c}0.7 \\
1 \\
\end{array}$ & $\begin{array}{c}0.79 * * \\
*\end{array}$ & $\begin{array}{c}0.74 * * \\
* \\
\end{array}$ & $\begin{array}{c}0.6 \\
8\end{array}$ & $\begin{array}{c}0.8 \\
1 \\
\end{array}$ & $\begin{array}{c}0.72 * * \\
*\end{array}$ & $\begin{array}{c}0.65^{* *} \\
* \\
\end{array}$ & $\begin{array}{c}0.6 \\
1 \\
\end{array}$ & $\begin{array}{c}0.6 \\
9 \\
\end{array}$ & $\begin{array}{c}0.76^{* *} \\
* \\
\end{array}$ & $\begin{array}{c}0.66^{* *} \\
*\end{array}$ & $\begin{array}{c}0.5 \\
9 \\
\end{array}$ & $\begin{array}{c}0.7 \\
3 \\
\end{array}$ \\
\hline $\mathrm{OBC}$ & $\begin{array}{c}0.79 * * \\
*\end{array}$ & $0.95 * *$ & $\begin{array}{c}0.9 \\
0 \\
\end{array}$ & $\begin{array}{c}1.0 \\
0 \\
\end{array}$ & $\begin{array}{c}0.80^{* *} \\
*\end{array}$ & $\begin{array}{c}0.91 * * \\
*\end{array}$ & $\begin{array}{c}0.8 \\
5 \\
\end{array}$ & $\begin{array}{c}0.9 \\
8 \\
\end{array}$ & $\begin{array}{c}0.80^{* *} \\
*\end{array}$ & 0.96 & $\begin{array}{c}0.9 \\
1 \\
\end{array}$ & $\begin{array}{c}1.0 \\
1 \\
\end{array}$ & $\begin{array}{c}0.72 * * \\
* \\
\end{array}$ & $\begin{array}{c}0.86^{* *} \\
*\end{array}$ & $\begin{array}{c}0.7 \\
9 \\
\end{array}$ & $\begin{array}{c}0.9 \\
4 \\
\end{array}$ \\
\hline Other & $\begin{array}{c}0.46 * * \\
* \\
\end{array}$ & $\begin{array}{c}0.68 * * \\
*\end{array}$ & $\begin{array}{c}0.6 \\
4 \\
\end{array}$ & $\begin{array}{c}0.7 \\
3 \\
\end{array}$ & $\begin{array}{c}0.50 * * \\
*\end{array}$ & $\begin{array}{c}0.69 \text { ** } \\
*\end{array}$ & $\begin{array}{c}0.6 \\
3 \\
\end{array}$ & $\begin{array}{c}0.7 \\
5 \\
\end{array}$ & $\begin{array}{c}0.46^{* *} \\
*\end{array}$ & $\begin{array}{c}0.70^{* * *} \\
* \\
\end{array}$ & $\begin{array}{c}0.6 \\
5 \\
\end{array}$ & $\begin{array}{c}0.7 \\
4 \\
\end{array}$ & $\begin{array}{c}0.47 * * \\
*\end{array}$ & $\begin{array}{c}0.72 * * \\
*\end{array}$ & $\begin{array}{c}0.6 \\
4 \\
\end{array}$ & $\begin{array}{c}0.8 \\
1\end{array}$ \\
\hline Religion & & & & & & & & & & & & & & & & \\
\hline Hindu ${ }^{\circledR}$ & & & & & & & & & & & & & & & & \\
\hline Muslim & $\begin{array}{c}0.79 * * \\
*\end{array}$ & $\begin{array}{c}1.11 * * \\
*\end{array}$ & $\begin{array}{c}1.0 \\
4 \\
\end{array}$ & $\begin{array}{c}1.1 \\
9 \\
\end{array}$ & 0.98 & $\begin{array}{c}1.32^{* *} \\
* \\
\end{array}$ & $\begin{array}{c}1.2 \\
0 \\
\end{array}$ & $\begin{array}{c}1.4 \\
4 \\
\end{array}$ & $\begin{array}{c}0.74 * * \\
* \\
\end{array}$ & $\begin{array}{c}1.10^{* * *} \\
*\end{array}$ & $\begin{array}{c}1.0 \\
3 \\
\end{array}$ & $\begin{array}{c}1.1 \\
8 \\
\end{array}$ & $\begin{array}{c}0.86^{* *} \\
* \\
\end{array}$ & $\begin{array}{c}1.33^{* *} \\
*\end{array}$ & $\begin{array}{c}1.1 \\
7 \\
\end{array}$ & $\begin{array}{c}1.5 \\
0 \\
\end{array}$ \\
\hline Other & $\begin{array}{c}0.72 * * \\
*\end{array}$ & $\begin{array}{c}0.78^{* *} \\
*\end{array}$ & $\begin{array}{c}0.7 \\
3\end{array}$ & $\begin{array}{c}0.8 \\
3\end{array}$ & $\begin{array}{c}0.76^{* *} \\
*\end{array}$ & $\begin{array}{c}0.77 \text { ** } \\
*\end{array}$ & $\begin{array}{c}0.7 \\
0\end{array}$ & $\begin{array}{c}0.8 \\
5\end{array}$ & $\begin{array}{c}0.68^{* *} \\
*\end{array}$ & $\begin{array}{c}0.74 * * \\
*\end{array}$ & $\begin{array}{c}0.6 \\
9\end{array}$ & $\begin{array}{c}0.7 \\
9\end{array}$ & $\begin{array}{c}0.85^{* *} \\
*\end{array}$ & 0.93 & $\begin{array}{c}0.8 \\
2\end{array}$ & $\begin{array}{c}1.0 \\
6\end{array}$ \\
\hline Wealth & & & & & & & & & & & & & & & & \\
\hline $\begin{array}{l}\text { Poorest } \\
\text { (B) }\end{array}$ & & & & & & & & & & & & & & & & \\
\hline Poorer & $\begin{array}{c}0.74 * * \\
*\end{array}$ & $\begin{array}{c}0.86^{* *} \\
*\end{array}$ & $\begin{array}{c}0.8 \\
1 \\
\end{array}$ & $\begin{array}{c}0.9 \\
1\end{array}$ & $\begin{array}{c}0.81 * * \\
*\end{array}$ & $0.92 * *$ & $\begin{array}{c}0.8 \\
5 \\
\end{array}$ & $\begin{array}{c}0.9 \\
9 \\
\end{array}$ & $\begin{array}{c}0.71 * * \\
*\end{array}$ & $\begin{array}{c}0.83 * * \\
* \\
\end{array}$ & $\begin{array}{c}0.7 \\
8 \\
\end{array}$ & $\begin{array}{c}0.8 \\
8 \\
\end{array}$ & $\begin{array}{c}0.69 * * \\
*\end{array}$ & $\begin{array}{c}0.79 * * \\
*\end{array}$ & $\begin{array}{c}0.7 \\
2 \\
\end{array}$ & $\begin{array}{c}0.8 \\
7 \\
\end{array}$ \\
\hline Middle & $\begin{array}{c}0.58 * * \\
*\end{array}$ & $\begin{array}{c}0.73 * * \\
*\end{array}$ & $\begin{array}{c}0.6 \\
8 \\
\end{array}$ & $\begin{array}{c}0.7 \\
7 \\
\end{array}$ & $\begin{array}{c}0.69 * * \\
*\end{array}$ & $\begin{array}{c}0.83^{* *} \\
*\end{array}$ & $\begin{array}{c}0.7 \\
6 \\
\end{array}$ & $\begin{array}{c}0.9 \\
0 \\
\end{array}$ & $\begin{array}{c}0.54^{* *} \\
*\end{array}$ & $\begin{array}{c}0.70^{* * *} \\
*\end{array}$ & $\begin{array}{c}0.6 \\
6 \\
\end{array}$ & $\begin{array}{c}0.7 \\
4 \\
\end{array}$ & $\begin{array}{c}0.57^{* *} \\
*\end{array}$ & $\begin{array}{c}0.71 * * \\
*\end{array}$ & $\begin{array}{c}0.6 \\
4\end{array}$ & $\begin{array}{c}0.7 \\
9 \\
\end{array}$ \\
\hline
\end{tabular}




\begin{tabular}{|c|c|c|c|c|c|c|c|c|c|c|c|c|c|c|c|c|}
\hline Richer & $\begin{array}{c}0.45^{* *} \\
*\end{array}$ & $\begin{array}{c}0.61 * * \\
*\end{array}$ & $\begin{array}{c}0.5 \\
6\end{array}$ & $\begin{array}{c}0.6 \\
5\end{array}$ & $\begin{array}{c}0.53^{* *} \\
*\end{array}$ & $\begin{array}{c}0.67 * * \\
*\end{array}$ & $\begin{array}{c}0.6 \\
1\end{array}$ & $\begin{array}{c}0.7 \\
4\end{array}$ & $\begin{array}{c}0.43^{* *} \\
*\end{array}$ & $\begin{array}{c}0.59^{* *} \\
*\end{array}$ & $\begin{array}{c}0.5 \\
5\end{array}$ & $\begin{array}{c}0.6 \\
3\end{array}$ & $\begin{array}{c}0.42 * * \\
*\end{array}$ & $\begin{array}{c}0.58^{* *} \\
*\end{array}$ & $\begin{array}{c}0.5 \\
1\end{array}$ & $\begin{array}{c}0.6 \\
6\end{array}$ \\
\hline \multicolumn{17}{|c|}{ Women Education Level } \\
\hline \multicolumn{17}{|c|}{ No Education $®$} \\
\hline Primary & $\begin{array}{c}0.83^{* *} \\
*\end{array}$ & 0.96 & $\begin{array}{c}0.9 \\
1\end{array}$ & $\begin{array}{c}1.0 \\
2\end{array}$ & $\begin{array}{c}0.83^{* *} \\
*\end{array}$ & 0.96 & $\begin{array}{c}0.8 \\
8\end{array}$ & $\begin{array}{c}1.0 \\
4\end{array}$ & $\begin{array}{c}0.83^{* *} \\
*\end{array}$ & 0.97 & $\begin{array}{c}0.9 \\
1\end{array}$ & $\begin{array}{c}1.0 \\
3\end{array}$ & $\begin{array}{c}0.87 * * \\
*\end{array}$ & 1.00 & $\begin{array}{c}0.9 \\
1\end{array}$ & $\begin{array}{c}1.1 \\
1\end{array}$ \\
\hline Higher & $\begin{array}{c}0.25^{* *} \\
*\end{array}$ & $\begin{array}{c}0.57 \text { ** } \\
*\end{array}$ & $\begin{array}{c}0.5 \\
1\end{array}$ & $\begin{array}{c}0.6 \\
3\end{array}$ & $\begin{array}{c}0.31 * * \\
*\end{array}$ & $\begin{array}{c}0.69^{* *} \\
*\end{array}$ & $\begin{array}{c}0.5 \\
9\end{array}$ & $\begin{array}{c}0.8 \\
1\end{array}$ & $\begin{array}{c}0.23^{* *} \\
*\end{array}$ & $\begin{array}{c}0.55^{* *} \\
*\end{array}$ & $\begin{array}{c}0.4 \\
9\end{array}$ & $\begin{array}{c}0.6 \\
1\end{array}$ & $\begin{array}{c}0.25^{* *} \\
*\end{array}$ & $\begin{array}{c}0.56 * * \\
*\end{array}$ & $\begin{array}{c}0.4 \\
5\end{array}$ & $\begin{array}{c}0.7 \\
1\end{array}$ \\
\hline \multicolumn{17}{|c|}{ Husband Education Level } \\
\hline \multicolumn{17}{|c|}{ No Education ${ }^{\circledR}$} \\
\hline Primary & $\begin{array}{c}0.86^{* *} \\
*\end{array}$ & 0.99 & $\begin{array}{c}0.9 \\
3\end{array}$ & $\begin{array}{c}1.0 \\
6\end{array}$ & $\begin{array}{c}0.85^{* *} \\
*\end{array}$ & 0.96 & $\begin{array}{c}0.8 \\
9\end{array}$ & $\begin{array}{c}1.0 \\
5\end{array}$ & $\begin{array}{c}0.87 * * \\
*\end{array}$ & 1.00 & $\begin{array}{c}0.9 \\
4\end{array}$ & $\begin{array}{c}1.0 \\
7\end{array}$ & $0.88 * *$ & 0.99 & $\begin{array}{c}0.8 \\
9\end{array}$ & $\begin{array}{c}1.1 \\
0\end{array}$ \\
\hline \multicolumn{17}{|c|}{ Husband Drink Alcohol } \\
\hline No ${ }^{\circledR}$ & & & & & & & & & & & & & & & & \\
\hline Yes & $\begin{array}{c}3.29 * * \\
*\end{array}$ & $\begin{array}{c}3.08 * * \\
*\end{array}$ & $\begin{array}{c}2.9 \\
6\end{array}$ & $\begin{array}{c}3.2 \\
1\end{array}$ & $\begin{array}{c}3.00 * * \\
*\end{array}$ & $\begin{array}{c}2.91 \text { ** } \\
*\end{array}$ & $\begin{array}{c}2.7 \\
5\end{array}$ & $\begin{array}{c}3.0 \\
7\end{array}$ & $\begin{array}{c}3.42 * * \\
*\end{array}$ & $\begin{array}{c}3.18^{* * *} \\
*\end{array}$ & $\begin{array}{c}3.0 \\
5\end{array}$ & $\begin{array}{c}3.3 \\
1\end{array}$ & $\begin{array}{c}3.37 * * \\
*\end{array}$ & $\begin{array}{c}3.15 * * \\
*\end{array}$ & $\begin{array}{c}2.9 \\
3\end{array}$ & $\begin{array}{c}3.3 \\
9\end{array}$ \\
\hline
\end{tabular}

\section{DISCUSSION}

There is a growing discussion on the high prevalence of intimate partner violence against women across the world which is resulting in reproductive and other health-related consequences (Watts \& Zimmerman, 2002; Campbell, 2002). There are studies suggesting that women are as aggressive as men in committing the violence against their partners (Archer, 2002; \& Straus, 1990) but this study relates to the violence against women inflicted by men. The causes of intimate partner violence are complex. Intimate partner violence cannot be attributed entirely to any one prime cause as it is the result of many causes. However, a few important factors and causes need to be focused. Research related to gender-based violence suggests that power relation between men and women is one of the critical factors of intimate partner violence, and men inflict violence against women to enforce their dominance and control over the women to uphold the cultural codes (Krishnaraj, 2007).

The key focus of this article is on studying the pattern, severity, and predictors of intimate partner violence. In this study, intimate partner violence describes emotional violence, physical violence, and sexual violence. The study found that with the increase in spousal age gap emotional violence increases. Physical and sexual violence first decreases with the increase in spousal age gap to an extent and then it increases with the increase in the spousal age gap. Sexual violence, emotional violence, and physical violence are most when the spousal age gap is more than 14 years. Physical violence is the most common and about 30 percent of women, in the spousal age gap ranging 0-4 years, have faced physical violence. In the same spousal age difference, about 13 percent of women have faced emotional violence, and 7 percent have faced sexual violence. Although prevalence captures only one dimension of the gender differences in the experience of spousal violence, but in no way, this can be misleading.

Intimate partner violence increases against the women with the increase in number of children ever born. This is because of the increase in time duration. As the number of children ever born increases, there is an increase in marital duration, and hence the chances of violence against women also increase. Straus et al., in their (2017) book based on the sample size of 2143 families in the United States found that the number of children is associated with the onset of intimate partner violence.

The socioeconomic characteristics also are very important in the discussion course of spousal violence against women. A higher percentage of women in Scheduled Caste (SC) and Scheduled Tribes (ST) face violence against them as compared to women in other caste group. Few studies in United States have found that women in minority ethnic group are more prone to face intimate partner violence (Dearwater et al., 1998 \& Jones et al., 1999). However backward classes in India and minority ethnic group in US are different but women in these groups are thought to be underprivileged. Similarly, a higher percentage of Hindu women face intimate partner violence than women of other religious group. Rural women as compared to urban women are more prone to face intimate partner violence, however there are studies that suggests that urban or rural residence are not the critical factors in determining the violence against women (Jewkes et al., 2002 \& Martin et al., 1999).

Poor women are more likely to face violence against them as compared to rich women. As the wealth quintile of household improves, the chances a female face violence decreases significantly. Jeyaseelan et al., (2007) based on 
their research carried out in rural, urban and urban slums of seven cities in India concluded that higher socioeconomic status acts as a protective buffer against spousal physical violence. The researchers are divided into two segment, the one who think that poverty has an effect on likelihood of violence against women (Martin et al., 1999; Raphael, 2001; Jewkes, 2002, Ratner, 1993 \& Ellsberg et al., 1999) and the other who think poverty as an insignificant factor in the occurrence of violence although after controlling variables like education and residence (Diop-Sidibe, 2001).

The above discussed two different ideologies of poverty and violence against women may have occurred as a result of large variability in defining household wealth, income, and socioeconomic status. Some researchers have used income (Rodgers, 1994), some have used information on assets (Diop-Sidibe, 2001 \& Martin et al., 1999), and others have used composite measure of socioeconomic status (Ellsberg et al., 1999) to define wealth.

Husband's Characteristics: It is husband who inflicts violence against his wife and so it is important to study husband's characteristics also. For the study, education level and alcohol consumption of the husband has been recorded. The study found that with the increase in the education level of husband, the chances a woman will face violence against her decreases significantly. The other studies also found that husband education level acts as a protective factor and women's whose husbands are educated are less likely to suffer from intimate partner violence (Naved \& Persson, 2005).

Similarly, those women whose husband consume alcohol are more prone to face intimate partner violence than those women whose husband do not consume alcohol. Alcohol consumption is associated with the increased risk of all forms of interpersonal violence (Farrington, 1998), and alcohol consumption by men is also found to be associated with intimate partner violence in many settings (Hoffman, Demo, \& Edwards, 1994; \& Kantor, 1993).

\section{LIMITATIONS OF THE STUDY}

Accurate and valid data on violence against women are not easy to collect. In a country like India where male takes privilege over the female in suppressing her identity, it is not easy to fathom the intensity of violence. The data on violence against women suffer from the bias as it is likely to be underreported in Indian context. Moreover, sexual violence has a very strong gender content and women may not open up completely and thus data on sexual violence suffer heavily from under-reporting. A study found that reported Gender-based violence rate in India is among the lowest in the world (Palermo et al., 2013).

\section{POLICY IMPLICATIONS}

Violence against women is one of the most widespread human rights and public health problem. Therefore, there is a need for a comprehensive response in order to curb the menace of violence against women. Based on the findings of this study, it is recommended that elimination of violence against women should remain the highest priority. Keeping in mind the under-reporting of data on violence, priority must be given to continued data collection and monitoring of violence against women. Comprehensive legislation on sex equality, intimate partner violence, and sexual violence should be advocated, per se, training and monitoring the police system shall be at place to ensure that legislation is satisfactorily enforced. Raising awareness through media must be the priority with the support from community actions and Non-Governmental Organisations. Empowering women and their status in society is another way to improve the statistics of violence cases against women. Empowerment can be taken through various channels like empowering women to control their fertility through various contraceptive services, by promoting gender equality in employment, by improving levels of female education, and by improving female's participation at local and national politics. At the same time, there is also need to address the men about the repercussions of violence against their wives.

\section{CONCLUSION}

The findings of this study draw attention to the pervasiveness of violence against women. There are numbers of reinforcing factors that increase the likelihood or severity of men's violence against women. Alcohol use by the husband is one of the prime causes of violence against women. The longer the marital duration, the higher is violence against women. Women accept the violence as their faith as they have no escape mechanism to it. Prevention of violence against women is still a visionary and transformative agenda. Identifying and addressing the cause of violence against women needs sustained efforts from individual as well as community-level along with proper governmental interventions. Increasing women's agency and mobilising women to claim their rights will also be helpful in combating the violence against them.

\section{REFERENCES}

[1] Agarwal, B., \& Panda, P. (2007). Toward freedom from domestic violence: The neglected obvious. Journal of Human Development, 8(3), 359-88.

[2] Ali, T. S., Asad, N., Mogren, I., \&Krantz, G. (2011). Intimate partner violence in urban Pakistan: prevalence, frequency, and risk factors. International journal of women's health, 3, 105.

[3] Archer, J. (2002). Sex differences in physically aggressive acts between heterosexual partners: A metaanalytic review. Aggression and Violent Behavior, 7, 213-351.

[4] Bamiwuye, S. O., \&Odimegwu, C. (2014). Spousal violence in subSaharan Africa: does household poverty-wealth matter?. Reproductive health, 11(1), 45.

[5] Bloom, T., Bullock, L. F., Sharps, P., Laughon, K., \& Parker, B. J. (2010). Intimate partner violence during pregnancy. In Family violence and nursing practice, 155-180. Springer Publishing Co, New York, NY.

[6] Bui, H. N., \&Morash, M. (1999). Domestic violence in the Vietnamese immigrant community: An exploratory study. ViolenceAgainst Women, 5(7), 769-795. 
[7] Campbell, J. C. (2002). Health consequences of intimate partner violence. The lancet, 359(9314), 1331-1336.

[8] Chin, K. (1994). Out of town brides: International marriage and wife abuse among Chinese immigrants. Journal of Comparative Family Studies, 25(1), 53-69.

[9] Dearwater, S. R., Coben, J. H., Campbell, J. C., Nah, G., Glass, N., McLoughlin, E., \&Bekemeier, B. (1998). Prevalence of intimate partner abuse in women treated at community hospital emergency departments. Jama, 280(5), 433-438.

[10] Devries, K. M., Kishor, S., Johnson, H., Stöckl, H., Bacchus, L. J., Garcia-Moreno, C., \& Watts, C. (2010). Intimate partner violence during pregnancy: analysis of prevalence data from 19 countries. Reproductive health matters, 18(36), 158-170.

[11] Diop-Sidibé, N. 2001. "Domestic Violence against Women in Egypt: Risk Factors and Health Outcomes of Wife Beating." Unpublished Ph.D. dissertation. School of Hygiene and Public Health, Johns Hopkins University.

[12] Ellsberg, M., Heise, L., Pena, R., Agurto, S., \&Winkvist, A. (2001). Researching domestic violence against women: methodological and ethical considerations. Studies in family planning, 32(1), 1-16.

[13] Farrington, D. P. (1998). Predictors, causes, and correlates of male youth violence. Crime and justice, 24, 421-475.

[14] Garcia-Moreno, C., \& World Health Organization. (2005). Multicountry study on women's health and domestic violence against women: initial results on prevalence, health outcomes and women's responses: Geneva, Switzerland.

[15] George, M. S., \&Rahangdale, L. (1999). Domestic violence and South Asian women. North Carolina Medical Journal, 60 (3), 157159.

[16] Hoffman, K. L., Demo, D. H., \& Edwards, J. N. (1994). Physical wife abuse in a non-Western society: an integrated theoretical approach. Journal of Marriage and the Family, 131-146.

[17] Hyder, A. A., Noor, Z., \&Tsui, E. (2007). Intimate partner violence among Afghan women living in refugee camps in Pakistan. Social Science \& Medicine, 64(7), 1536-1547.

[18] Jejeebhoy, S. J. (1998). Wife-beating in rural India: a husband's right? Evidence from survey data. Economic and Political weekly, 855-862.

[19] Jewkes, R. (2002). Intimate partner violence: causes and prevention. The lancet, 359(9315), 1423-1429.

[20] Jewkes, R., Levin, J., \& Penn-Kekana, L. (2002). Risk factors for domestic violence: findings from a South African cross-sectional study. Social science \& medicine, 55(9), 1603-1617.

[21] Jeyaseelan, L., Kumar, S., Neelakantan, N., Peedicayil, A., Pillai, R., \&Duvvury, N. (2007). Physical spousal violence against women in India: some risk factors. Journal of biosocial science, 39(5), 657-670.

[22] Jones, A. S., Gielen, A. C., Campbell, J. C., Schollenberger, J., Dienemann, J. A., Kub, J., \& Wynne, E. C. (1999). Annual and lifetime prevalence of partner abuse in a sample of female HMO enrollees. Women's Health Issues, 9(6), 295-305.

[23] Kantor, G. K. (1993). Refining the brushstrokes in portraits of alcohol and wife assaults. Alcohol and interpersonal violence: Fostering multidisciplinary perspectives, 281-290.

[24] Krishnaraj, M. (2007). Understanding violence against women. Economic and Political Weekly, 42 (44), 90-91.

[25] LaRusso, L. (2016). Intimate Partner Violence During Pregnancy. Nursing for Women's Health, 20(3), 239.
[26] Leonard, K. E. (2005). Alcohol and intimate partner violence: when can we say that heavy drinking is a contributing cause of violence?. Addiction, 100(4), 422-425.

[27] Martin, S. L., Tsui, A. O., Maitra, K., \&Marinshaw, R. (1999). Domestic violence in northern India. American journal of epidemiology, 150(4), 417-426.

[28] Naved, R. T., \&Persson, L. A.. (2005). Factors associated with spousal physical violence against women in Bangladesh. Studies in family planning, 36(4), 289-300.

[29] Palermo, T., Bleck, J., \& Peterman, A. (2013). Tip of the iceberg: reporting and gender-based violence in developing countries. American journal of epidemiology, 179(5), 602-612.

[30] Panda, P., \&Agarwal, B. (2005). "Marital violence, human development and women's property status in India." World Development, 33 (5), 823-50.

[31] Perilla, J. L. (1999). Domestic violence as a human rights issue: The case of immigrant Latinos. Hispanic Journal of Behavioral Sciences, 21 (2), 107-133.

[32] Raj, A., \& Silverman, J. G. (2002). Intimate partner violence against South Asian women in greater Boston. Journal of the American Medical Women's Association (1972), 57(2), 111-114.

[33] Raphael, J. (2001). Public Housing and Domestic Violence. Violence Against Women, 7, 699-706.

[34] Ratner, P. A. (1993). The incidence of wife abuse and mental health status in abused wives in Edmonton, Alberta. Canadian journal of public health= Revue canadienne de santepublique, 84(4), 246-249.

[35] Rodgers, K. 1994. "Wife Assault: The Findings of a National Survey." Juristat Service Bulletin 14(9), 1-22.

[36] Schuler, S. R., Hashemi, S. M., Riley, A. P., \&Akhter, S. (1996). Credit programs, patriarchy and men's violence against women in rural Bangladesh. Social science \& medicine, 43(12), 1729-1742.

[37] Silverman, J. G., Gupta, J., Decker, M. R., Kapur, N., \& Raj, A. (2007). Intimate partner violence and unwanted pregnancy, miscarriage, induced abortion, and stillbirth among a national sample of Bangladeshi women. BJOG: An International Journal of Obstetrics \& Gynaecology, 114(10), 1246-1252.

[38] Straus, M. A., Gelles, R. J., \& Steinmetz, S. K. (2017). Behind closed doors: Violence in the American family. Routledge.

[39] Straus, M.A. 1990. Measuring intrafamily conflict and violence: The conflict tactic (CT) scales. In M.A. Straus and R.J. Gelles (Eds.) Physical Violence in American families: Risk Factors and Adaptations to Violence in 8,145 Families, 29-47. New Brunswick: Transaction Publishers.

[40] Varma, D., Chandra, P. S., Thomas, T., \& Carey, M. P. (2007). Intimate partner violence and sexual coercion among pregnant women in India: relationship with depression and post-traumatic stress disorder. Journal of Affective Disorders, 102(1-3), 227-235.

[41] Watts, C. and C. Zimmerman. 2002. Violence against women: Global scope and magnitude. The Lancet, 359, 1232-1237.

[42] World Health Organization. (2011). Intimate partner violence during pregnancy: Information sheet.

[43] World Health Organization. (2012). Understanding and addressing violence against women: intimate partner violence. 